\title{
Optimisation of Online Newspaper Headline Length with Characters
}

\author{
${ }^{1}$ Nasibu M.K Musa*, ${ }^{1}$ Dr. Gechemba D. Nyakoe, ${ }^{2}$ Dr. Kenneth Odhiambo \\ ${ }^{1}$ Department of Languages, Linguistics and Literature, Rongo University, Kenya \\ ${ }^{2}$ Department of Languages, Linguistics and Literature, Maasai Mara University, Kenya \\ *Corresponding author: musanasibu@yahoo.com
}

\begin{abstract}
The question of how many characters should an online newspaper headline have is still debatable. This study therefore, sought to examine the optimal length for the online newspaper headlines by using characters as the factor for online news readership. In an attempt to establish an optimal headline length, a sample of 259 out of 730 headlines published by the Tanzanian newspapers of Mwananchi and The Citizen from July 2017 to June 2018 was collected. The study employed Coschedule to obtain the number of characters for each online newspaper headlines. The data were quantitatively analysed with SPSS version 20. The study observed that $72.2 \%$ of 259 headlines that Mwananchi and The Citizen digital journalists designed were short ranging from 16 to 45 characters long on the average. The study accepted the null hypothesis that there was no significant statistical difference in readership between short and long online newspaper headlines. This finding was against the long-standing print newspaper assumption that newspaper readers prefer short headlines to the long ones. The study recommends other studies on the same topic to have a comparative study on headline readership across search engines, social platforms and languages.
\end{abstract}

Keywords: Online Newspaper headlines, length, characters, optimization, readership

\section{Introduction}

In the past decades, digital journalists have tried to suggest different optimal lengths for online posts. However, the ideal online newspaper headline length is still controversial (Morgan, 2017). In the contemporary newspaper industry, the question of the headline length is explained in two principle views (Neidlinger, 2015).The first and traditional view as proposed by Holmqvist et al. (2003), Nielsen (2009), Dor (2003) and Saxena (2006) is that a headline should be as short as possible because online news readers scan more and read less than their counterparts print newspapers readers do. In this view, a newspaper headline ought to be rich in content and act as an abstract of the article introduced. The second view as stated by Nielsen (2009) is that a headline should be somewhat longer, confusing, bizarre, shared frequently on the social platforms and that which receives high ClickThrough Rates (CTR) on the web. In this school of thought, what matters is not an information-rich headline but an emotion-rich headline that can have repeated shares on the social platforms. Based on this controversy, this paper therefore sought to address the question of the ideal length of the online newspaper headline regarding the number of characters it contains by using the data from the websites of Mwananchi and The Citizen newspapers in Tanzania.

\section{Determinants of the Ideal Length of a Headline}

In the world of content marketing and the mass communication fields, a lot of attention has been paid to the online headline length (Neidlinger, 2015). This is because the best headline normally gets the most attraction for social traffic, shares and ranking in the search engines. Different studies have tried to determine headlines in several ways. In most cases, the headline length can be determined by variables such as the number characters, syllables and words. These variables are also the standard measures of a headline length as they determine web ranking and visibility in Search Engine Optimization (SEO). Dor (2003), Saxena (2006), Lee, (2014) and Kuiken et al., (2017) have used the number of words, syllables and characters 
as the factors for headline length. Just like the previous studies, this study delimited itself to the number of characters as the standard measure of the optimal newspaper headlines. Even though there are so many free online calculators such as Microsoft Office Word 97-2019, AbiWord, and WordPerfect, which can count the number of characters in newspaper, Facebook, Twitter posts, as well as other online posts, CoSchedule character counts are pretty simple features and quite useful (Krumholz, 2016). According to Krumholz, this online headline analyzer recommends whether the digital newspaper designer should shorten or lengthen their headlines regarding the standards of about 55 characters. It was in light of this CoSchedule standard character, that the best newspaper headline for SEO was defined under this study.

\section{Newspaper Headlines as the Relevance Optimisers}

This study was based on Sperber and Wilson's theory of relevance $(1986,1995$, Wilson \& Sperber, 2002). Under this theory, the study made a claim that newspaper headlines are relevance optimisers. Based on this fact, the digital journalists tend to optimise relevance of their headlines with characters as the factor for search engine optimisation, visibility and web ranking. According to the Cognitive Principle of relevance theory, human cognition is geared to the maximisation of relevance. According to Dor (2003), newspaper headlines are relevance optimisers. Therefore, journalists can maximize the relevance of the headlines with the ideal number of characters. They assess the relevance of their newspaper headlines by considering the cognitive effects and processing efforts. According to Wilson and Sperber (2002 p. 250), "Other things being equal, the greater the positive cognitive effects achieved by processing an input, the greater the relevance of the input to the individual at that time." Based on this quotation, a newspaper headline length was said to be relevant to the readers when it had greater positive cognitive effects and less processing efforts. Thus, the headline designers had to select the ideal headline length based on characters to reduce the processing efforts and maximize the positive cognitive effects. Wilson and Sperber (2002) also say, "Other things being equal, the greater the processing effort expended, the lower the relevance of the input to the individual at that time (p.250). This means that a headline was irrelevant to readers when it required greater processing efforts to achieve the maximum cognitive effects.

Based on the traditional assumption by Dor (2003), a short headline is said to be more relevant than the short one because it is easier to comprehend. In search engines, the online headlines have a limited number of characters that they can easily display in the search outputs (Dwyer, 2016). Thus, a headline can be irrelevant to readers if it exceeds the specified number of characters allowed in a search engine and relevant when it is within the allowed number of characters. Newspaper headline designers, therefore, should consider the optimum number of characters allowed by the search engines if they wish news headlines to display well in the search outputs.

In the ostensive inferential communication of the Communicative Principle, however, utterances create expectations of optimal relevance (Sperber and Wilson 1986). Under this principle, there are two intentions: Intention to inform the audience of something and intention to communicate. In this regard, the online headline designers normally intend to inform and their readers expect the headlines published to be informative. To achieve their communicative goals, the online headline designers show their communicative intentions by creating headlines with the ideal number of characters to allow their readers to search the news with ease on the internet. So, characters become the overt strategies that online journalists use to facilitate information search and readership on the internet.

\section{Headline Characters and Search Engine Optimisation}

Nowadays, search engines such as Google, Bing and Yahoo are increasingly becoming part of people's everyday life especially when they are looking for information they want. This is because news seekers are hooked on the above mentioned search engines to access the information they want. Dwyer (2016) highlights the 12 top search engines or browsers in the world, which are Google, the biggest and most popular search engine in the world by 2015, Bing, the Microsoft's default Web Browser), Yahoo, Baidu , the popular browser in China), AOL, Ask.com, Excite, DuckDuckGo, Wolfram Alpha, Yandex, Lycos and Chacha.com. Each of these search engines has a limited number of characters that it can easily display in its search output. Thus, the headlines that meet the standard number of characters are 
expected to have the best performance on the search engine. Newspaper headline designers, therefore, need to consider the relevant number of characters per search engine if they wish their news headlines to display well in the search engines.

In light of Search engine Optimization (SEO), headlines are title tags for newspaper pages that are visible in the search results and they are therefore significant in determining news readership. However, there are different conflicting views about a page headline that is recommended in search results pages (SERPS). This is because each search engine has a limited number of characters (Rogers, 2013). Rogers claims that Yahoo recommends a title page length with the maximum of 67 characters, Bing recommends a headline of about 65 characters, W3C recommends 64 characters and Google has no guidance on its headlines. Since headlines are relevancy optimisers (Dor, 2003), this study claimed that Mwananchi and The Citizen online newspaper headline designers ought to select the ideal length (characters) for their headlines if they wish to increase headlines visibility and ranking on the search engines. So, this paper was an attempt to make a claim that there is an ideal length for online newspaper headlines.

\section{Literature Review}

Several studies have been done on the ideal headline length regarding the characters in different search engines. Some studies have attested that the ideal number character per headline varies across for search engines and social platforms. This section, therefore, presents some literature on the ideal number of characters for online posts. To begin with, Lake's (2011) study revealed that a headline for Google News should range from 65 to 70 characters and Yahoo should be 120 characters. According to Lake, the character limitation is based on the fact that search engines such as Google tend to truncate long headlines of more than 70 characters. In Lake's study, for example, it was reported that $77 \%$ of 15,000 Google News headlines with more than 70 characters long were truncated. In light of this fact, headline length was a very important factor for online readership of the selected online newspaper headlines. Therefore, the writers of Mwananchi and The Citizen headlines had to choose headlines with a limited number of characters to avoid truncation in different search engines.
Some studies have also shown that the ideal length of an online newspaper headline varies for online social platforms, blogs and websites. Ashwell (2013), who used Hubspot marketing software to study the impact of headline length on Facebook shares, proposed that the perfect headline was 80 and 130 or 160 characters long, including spaces. Loranger and Nielsen (2017) on the other hand have maintained that the best headlines for news sites should be very short (less than 40 characters long). Lee's (2014) study, in contrast, proposed that a blog headline of fewer than 50 characters is likely to perform well on the digital setting. On the social networks, Lee (2015) discovered that the ideal length for a Twitter post was 71-100 characters, the Facebook post was 40 characters, Google was more than 40 characters and Linkedln was 80-120 characters. In light of this observation, it is obvious that the performance of any post relies heavily on the social media. Contrary to these studies, the current paper had its focus on the ideal length of online newspaper headlines.

Hubspot and Outbrain's (2015) survey of 100,000 headlines posted online proposed 80 to 110 characters as the ideal length for driving engagement. CoSchedule (2015) on the other hand suggested a headline with about 50 characters long. The studies by Ashwell (2013), CoSchedule, (2015), Lee (2014) and Hubspot and Outbrain (2015) are good testimonies that the views on the ideal length of online newspaper headlines are still disputed. The other study by Hubspot and Outbrain (2015) has shown that there are different ideal characters in different languages. It was shown that the best post for English headlines was 60-70 characters. That is, any English headline with more or less than 70 characters would experience the reduced Click through Rate (CTR).

Moreover, Hubspot and Outbrain's study observed that Italian and French headlines flared equally to the English headlines. Nevertheless, Spanish Ionger headlines outperformed the shorter ones. The study concluded that the ideal length for headlines was not similar for every language and culture. Thus, the ideal headline length for English newspaper sites in the USA may not be similar to a Swahili site in Tanzania. That is why this study focused on the ideal length for headlines in the newspapers. Despite the disparity in the number of characters that a headline should have, the above studies seem to agree that there should be an optimum length for online publication that varies for search engines, social 
media, language, and culture. This study, therefore, focused on establishing the ideal newspaper headlines for English and Swahili online publications.

\section{Methodology of the Study}

This study adopted the analytical research designs, which is associated with both qualitative and quantitative approaches. This study involved a population of 10 online newspapers in Tanzania and a sample of two dailies (Mwananchi and The Citizen), which were published by Mwananchi Communication Limited (MCL). The study used a sample of 259 out 730 front-page newspaper headlines published from 2017 July to June 2018. The MCL dailies were purposively sampled for they were the Tanzanian online newspapers that had a good record of online newspaper readership metrics. Since the ideal headline length varied for the social media, search engine, language and culture (Hubspot \& Outbrain, 2015), Mwananchi, which was the Swahili paper was purposely picked to represent the majority of readers in Tanzania who could speak and read Swahili news. The Citizen was also sampled for English audience. Mwananchi and The Citizen were the quality newspapers with a wide content coverage and readership in Tanzania (Khalifa, 2018 \& MCT, 2018). Besides, the two daily newspapers from the same company, which were the part of Nation Media Group of Kenya (NMG), had a good record of online specific newspaper headline readership metrics in Tanzania. In this regard, the study had no option rather purposively choosing the MCL daily newspapers.

The paper used Yamane's (1967) sample formula to obtain a sample of 259 headlines out of 730 . The confidence level chosen was 95\%, which was equivalent to a 0.5 significance level. The $95 \%$ level of accuracy in sampling is always adequate to make the optimal sample size (Yamane, 1967). Therefore, this confidence level was expected to give reliable study results. The study employed the ComputerBased Systematic Random-Sampling program to obtain the 259 newspaper headlines from 730 headlines. This was equivalent to 129.5 headlines for each newspaper from July 2017 to June 2018. In this study, $50.2 \%$ of 259 sampled newspaper headlines came from Mwananchi and $49.8 \%$ came from The Citizen headlines. This evidences that the two online papers had almost equal representation in the population of the study. Thus, the study sample was enough to conclude on the ideal length of an online newspaper headline.

\section{Data Collection Tools}

This study employed a checklist to get data from Mwananchi and The Citizen. The checklist contained the headline codes ranging from 1 to 259 for SPSS analysis. Secondly, there were newspaper codes (1 = Mwananchi, 2 =The Citizen); the year of publication codes $(1=2017$ or $2=2018)$, and the month of publication (July 2017 and June 2018) codes. The other item in the checklist was set to track headlines length in terms of characters. For the sake of SPSS menu and convenient analysis, the number of characters per headline had to be captured within five variables in the interval scales of $1=(0-15$ characters), $2=(16-30$ characters $), \quad 3=(31-45$ characters), $4=(46-60$ characters $)$ and $5=(60+$ characters).

The other item was set to capture the readership metrics variables (1= number of clicks, $2=$ number of views). The number of clicks was defined as the number of people who clicked or opened the web page containing the headline whereas the number of views defined as the number of people who happened to visit the page. CTR formula was used to test whether the number of characters in the headline had a significant impact on the newspaper readership on the internet or not. The study used CTR formula because it is the most reliable and commonly used metric for evaluating the performance and readership of internet documents (Konig, Gamon, \& Wu, 2009; Richardson, Dominowska \& Ragno, 2007).

Finally, Neidlinger (2015) automatically calculated the number of characters per headline and recommended whether the headline had to be lengthened or shortened. To determine the relationship between headline length regarding characters and readership, two hypotheses below (null and alternative) were developed:

H1: Online newspaper readers prefer shorter headlines ( $\leq 55$ characters) over longer headlines (>55 characters)

$\mathrm{HO}$ : There is a similar preference in readership between longer headlines (>55 characters) and shorter headlines ( $\leq 55$ characters).

The numbers of characters in intervals scales were coded purposely to get the intended information. In coding, they were defined with label values $(1=1$ 15 char.), ( $2=16-30$ char.), ( $3=31-45$ char.), ( $4=46-$ 60 char.), and ( $5=61+$ char.) whereas the CTR's assessments values were ( 1 = very low readership), 
(2 = low readership), ( $3=$ average readership), (4 = high readership) and 5 =very high readership). Concerning the hypothesis, there was a need to focus on the variation among group means $(\leq 55$ characters and $<55$ characters). By considering the nature of the research data, a one-way ANOVA fitted for the analysis because of the dependent variables that were in intervals. The independent variable (characters) consisted of two or more categories. In this regard, there was an independent observation, a normal distribution of independent variables and homogeneity of variance testing through Laverne's Test.

\section{Findings and Discussion}

The results under this study were based on how the digital journalists of Mwananchi and The Citizen optimized relevance of their headline with characters and their impact to online newspaper readers.

\section{Character Preference by Newspaper Headline Writers}

This paper sought to confirm whether online newspaper writers should optimise online headlines to enable readers search for information published online. By doing so, the headlines would get more engagements on the web. According to Sperber and Wilson (1986/1995), newspaper headlines are relevance optimisers. Thus, the headline writers or editors should optimise relevance of their headlines by creating headlines with about 55 characters if they want their posts to go viral on the social platforms. After analysing 259 newspaper headlines, the results on headline length preference by journalists from Mwananchi and The Citizen showed that $21.6 \%$ out of 259 sampled headlines contained 16 to 30 characters, $55.6 \%$ contained 31 to 45 characters, $20.1 \%$ contained 46 to 60 characters, and $2.7 \%$ contained more than 61 characters. Thus, the finding shows that Mwananchi and The Citizen journalists preferred headlines with 31 to 45 characters. Mwananchi and The Citizen newspaper writers were still observing the traditional print media in designing short (31-45) headlines for digital publications. After looking at printed newspaper headlines; it was revealed that most of the print and online newspapers were identical. Figure 1 presents the number of characters from Mwananchi and The Citizen headlines.

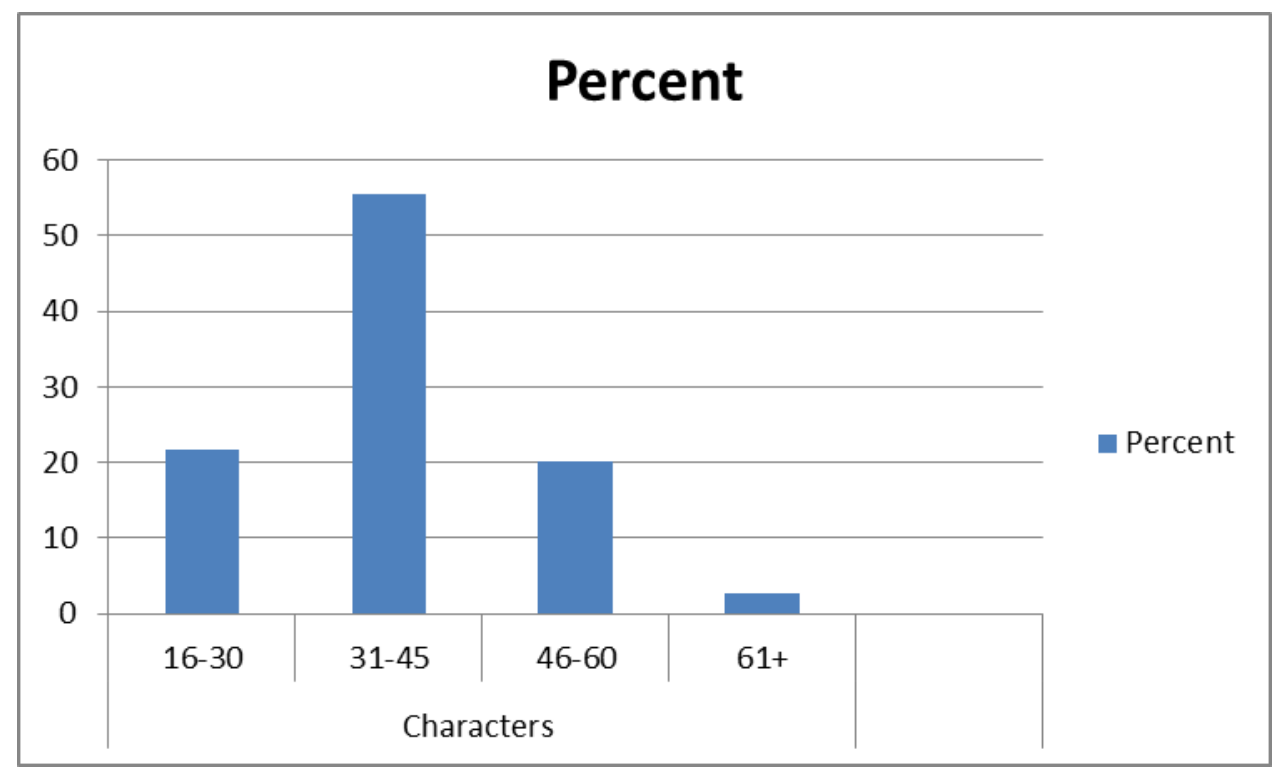

Figure 1: Number of Characters per Digital Newspaper Headline

The data set in $a, b$ and c examples presents the samples of newspaper headlines with varying degrees of characters.

a. Japanese teachers visit Tanzania schools. [36 char.] (Namkwahe, 2017). b. Government asked to supervise allocations of abandoned farms [53 char.] (Shariff, 2017).

c. Tanzania lawmakers concerned as ExxonMobil plans to sell out of gas field. [63 char.] (Kamndaya, 2018). 
The first headline had 36 characters, the second one had 53 characters and the third one had 63 characters. According to CoSchedule, the online headline analyser software, headlines with about 55 characters long tend to earn the highest number of click-through rates (CTR). By referring to the CoSchedule rule, only $21 \%$ ( $46-50$ characters length) of all 259 headlines published by The Citizen and Mwananchi would earn the highest CTR. Since Lee (2014) argues that the ideal length of a Tweet post is 71-100 characters, the finding in this study shows that only $2.7 \%$ of Mwananchi and The Citizen newspaper headlines would perform well when posted and shared on Twitter. According to Bullas (2013), the Facebook posts with ultra-short 40 characters receive $86 \%$ higher engagement. In this regard, $72.2 \%$ of the newspaper headlines studied would perform better on Facebook. Farnsworth (2012) on the other hand claims that headlines should not exceed 60 characters on Google and 80100 on Linkedln. In this regard, $97 \%$ of all 259 newspaper headlines studied would perform better on Google search engine and 2.7 would have more shares on Linkedln. Based on the previous studies, it can be concluded that most headlines of the two newspapers would perform well on Google search engine but poorly on social media such as Twitter and Linkedln.

In relevance theoretical framework by Sperber and Wilson (1986), newspaper headlines are relevance optimisers. Thus, the digital journalists designed relevant newspaper headlines by considering the audience's processing efforts and cognitive effects. They tried to maximize the relevance of newspaper headlines with the limited number of characters and consideration that headlines are truncated when they are above limits of search engines. The best headline would display the best search results in the browser such as Google. In Ostensive inferential communication, the headline designers intended to inform the audience and the informative intention is seen through communicative headlines with ideal characters for search engines.

\section{The Ideal Characters for Online Newspaper Headlines}

The study also sought to examine how the number of characters per headline affected news readership on the selected newspapers' website. The ANOVA analysis on significance in table 1 shows that there was no statistically significant difference in readership between longer headlines (>55 characters) and shorter headlines ( $\leq 55$ characters) since $F(3,255)=3.583, P=0.194$ and $\alpha=0.05$ at twotailed. Thus, the study accepted the null hypothesis that there was a similar preference in readership between longer headlines ( $>55$ characters) and shorter headlines $<55$ characters. The observed finding proposed that headlines length (characters) was not the factor that determined a click-through rate (readership) because headlines of different degrees of lengths [16-35 \& 61+ char.) had similar click-through rates.

Table 1: Analysis of Variance

\begin{tabular}{|c|c|c|c|c|c|}
\hline & Sum of Squares & d.f & Mean Square & $\mathbf{F}$ & Sig. \\
\hline Between Groups & 10.775 & 3 & 3.592 & 1.583 & 0.194 \\
\hline Within Groups & 578.414 & 255 & 2.268 & & \\
\hline Total & 589.189 & 258 & & & \\
\hline
\end{tabular}

Therefore, there is no ideal number of characters for newspaper headline because short headlines (31-45 characters) and long headlines (61+characters) received similar number of clicks.

\section{Conclusions and Recommendations}

This part gives the conclusions and recommendations of the study.

\section{Conclusions}

It is concluded that Mwananchi and The Citizen journalists prefer headlines with 31 to 45 characters. They are still observing the traditional print media in designing short headlines (31-45 characters) for digital publications. In other words, they optimized relevance of their headlines with relatively short headlines. However, there is no significant difference between the number of characters that the headline contains and online newspaper headline readership. Despite the digital journalists' preference on short headlines (31-45 characters), the analysis of headline readership web metrics with ANOVA showed that both short headlines (16-30 char.) and long headlines (61+ char.) were equally preferred by readers on the 
internet. Therefore, both short and long online newspaper headlines can have similar impact on news readership.

\section{Recommendations}

Since this study was based on characters as the variable for headline length optimization, other studies on online headline length should focus on other variables such as syllables and words in order to figure out the ideal headline length across search engines, social platforms and languages. In light of headline performance, a comparative study on the impact of a headline length on news readership across browsers such as (Yahoo, Bing and Google), social platforms (Facebook, Instagram and Twitter) and world languages is highly recommended.

\section{Reference}

Ashwell, R. (April $8^{\text {th }}, 2013$ ). How long is a perfect headline? Apparently 80,130 or 160 characters? Publitek. Retrieved from https://www.publitek.com/news/how-longis-a-perfect-headline-apparently-80-130-or160-characters/

Bullas, J. (2013). 21 Awesome social media facts, figures and statistics for 2013. Blog Post. Retrieved in December 2019 from https://smallbusiness. yahoo.com/advisor/2 1-awesome-social-media-facts-figuresstatistics-2013-231748416.htm.

Coschedule (2015). Free Headline Analyzer (Online software). Retrieved from https://coschedule.com/headline-analyze

Dor, D. (2003).On newspaper headlines as relevance optimizers: Journal of Pragmatics. 35 (5), 695-721.

Dwyer, D. (2016). Top 12 best search engines in the world. Retrieved January, 29, 2019 from https://scholar.google.com/scholar?hl=en\& as_sdt=0\%2C5\&q=Top+12+Best+Search+Eng ines+in+The+World\&btrnG $=$

Farnsworth, D. (2012, September 18). Reasons Why Every Web Designer Needs to Learn Rails. Retrieved on 5th August 2019 from http://blog.team treehouse. Com/reasonswhy-every-web-designer-needs-to-learnrails.

Holmqvist, K., Holsanova, J., Barthelson, M., \& Lundqvist, D. (2003). Reading or scanning? A study of newspaper and net paper reading.
In The Mind's Eye (pp. 657-670). NorthHolland.

Hubspot and Outbrain (2015) "Data driven strategies for writing effective titles \& headlines" Retrieved from http://cdn2.hubspot.net/hub/53/file250555691

Kamndaya, S. (2018, June 19). Tanzanian lawmakers concerned as ExxonMobil plans to sell out of gas field. The Citizen. Retrieved from https://www.thecitizen.co.tz/news/184034 0-4620274-5fpa01z/index.html

Khalifa, S. (2018, November 15). The Citizen Newspaper publisher named champion in Tanzania's print media market study. The Citizen. Retrieved from https://www.thecitizen.co.tz/news /184034 0-4853370-4an33qz/index.html

König, A. C., Gamon, M., \& Wu, Q. (2009, July). Clickthrough prediction for news queries. In Proceedings of the 32nd international ACM SIGIR conference on Research and development in information retrieval (pp. 347-354). ACM.

Krumholz, M. (2016, October 18). How to write effective headlines with CoSchedule's Headline Analyzer. Verblio Blog: Retrieved from https://www.verblio.com/blog/writeeffective-headlines-coschedule

Kuiken, J., Schuth, A., Spitters, M., \& Marx, M. (2017). Effective headlines of newspaper articles in a digital environment. Digital Journalism, 5(10), $1300-1314$. Retrieved fromhttps://doi.org/10.1080/

21670811.2017.1279978.

Lake, C. (2011, October 27). How to optimise headlines using the 65-character rule. Econsultancy Blog. Retrieved from https://econsultancy.com/how-to-optimisehe.

Lee, K. (2014). The proven ideal length of every tweet, Facebook post, and headline online. Fast Company, Apr. Accessed on https://www.fastcompany.com/3028656/th e-proven-ideal-length-of-every-tweetfacebook-post-and-headline-online. 
Loranger, H., \& Nielsen, J. (2017). Microcontent: A Few Small Words Have a Mega Impact on Business. US: Nielsen Norman Group.

Morgan, A. (2017, May 24). The ideal length of a headline: Are short headlines more effective? Retrieved from https://magazine.vunela.com/the-ideallength-of-a-headline-175c5db31a44

Neidlinger, J. (2015, February 16). What really is the best headline length? CoSchedule Blog. Retrieved from https://coschedule. com/blog/best-headline- length/

Nielsen, J. (2009, April 26). World's best headlines: BBC News. Nielsen Norman Group. Retrieved from https://www.nngroup.com/articles/worldsbest-headlines-bbc-news

Richardson, M., Dominowska, E., \& Ragno, R. (2007, May). Predicting clicks: estimating the clickthrough rate for new ads. In Proceedings of the 16th International Conference on World Wide Web (pp. 521-530). ACM.
Rogers, R. (2013). Digital methods. Cambridge, MA: MIT press.

Saxena, S. (2006). Headline writing. Delhi: SAGE Publications India.

Shariff, H. (2017, August 29). Govt asked to supervise allocations of abandoned farms. The Citizen. Retrieved from https://www.thecitizen.co.tz/news/Govtasked-to-supervise-allocations-ofabandoned-farms/1840340-4075842format-xhtml-aaiv7v/index.html

Sperber, D \& Wilson, D. (1986). Relevance: Communication and Cognition. Vol.142. Oxford: Blackwell.

Sperber, D \& Wilson, D. (1995). Relevance: Communication and Cognition. Oxford: Blackwell.

Wilson, D. \& Sperber, D. (2002) Truthfulness and relevance. Mind, 111. 583-632.

Yamane, T. (1967) Statistics: An introductory analysis, Second edition. New York: Harper and Row. 